\title{
Study on Lubrication Flow and Heat Transfer Characteristics of Under-Race Lubrication for High Speed Ball Bearing
}

\author{
Yuxiang FENG, Jinyu YAO ${ }^{1}$, Xiaohan SUN and Cunqing YUAN \\ College of Aerospace and Civil Engineering, Harbin Engineering University, Harbin \\ 150001, China
}

\begin{abstract}
In order to reveal the lubrication characteristics of high-speed ball bearings under the sub-ring lubrication mode and improve the lubrication efficiency of ball bearings, a three-dimensional geometric model of high-speed ball bearings was established. By using multiple reference frame and VOF model, the two-phase flow field and temperature field of inner and outer ring in the bearing under different conditions were investigated, and the correlation law of lubricating oil flow and heat transfer was obtained. At the same time, the definition of the penetration rate was given quantitatively for the lubrication efficiency of the outer ring. The lubrication efficiency of ball bearings under-race lubrication was evaluated from the aspects of rotation speed and penetration rate. The results showed that the oil and gas distribution and temperature distribution in the lubrication under the ring are not uniform, and there was an obvious correlation between the two; the increase of speed reduced the oil volume fraction in the bearing. The oil distribution was more uniform which was useful for reducing the temperature difference between the inner and outer rings.
\end{abstract}

Keywords. Ball bearing, under-race lubrication, oil and gas phases, lubrication performance, numerical simulation

\section{Introduction}

Modern advanced gas turbine engines are developing in the direction of high thrust-toweight ratio, low fuel consumption, high reliability, and long life [1], which makes the working conditions of the main shaft increasingly harsh. At present, the $\mathrm{DN}(\operatorname{Speed}(\mathrm{r} / \mathrm{min}) \times$ Inner diameter $(\mathrm{mm}))$ value of the main bearing of the aero-engine has reached $2 \times 10^{6} \sim 3.5 \times 10^{6} \mathrm{DN}$ or more [2]. With the continuous increase of the bearing speed, the gas flow in the bearing cavity becomes more and more intense, and a highspeed cyclone will be generated near the ball, forming a high-pressure zone, hindering the delivery of lubricating oil to the contact area [3]. Therefore, to achieve the lubrication function more efficiently and reduce the harmful centrifugal force generated under the high DN value, the under-ring lubrication technology has been developed [4]. The lubricating oil can enter the bearing through the groove and radial hole on the inner ring to realize the lubrication and cooling of the bearing balls [5]. NASA took the lead in conducting a large number of experimental studies on under-ring lubrication technology

\footnotetext{
${ }^{1}$ Corresponding Author, Jinyu YAO, College of Aerospace and Civil Engineering, Harbin Engineering University, Harbin 150001, China; E-mail: yaojinyu@hrbeu.edu.cn
} 
in the early 1970s [6-7], and obtained the changing rules of the under-ring lubrication bearing operating performance under different working conditions, which provided reliable lubrication mechanism optimization in accordance with. Affected by the internal structure and contact characteristics of the bearing, and the relative movement of the components, many experiments cannot meet the research needs. Numerical calculations have become a powerful tool for studying the flow and distribution of the lubricating medium in the bearing [8]. Generally speaking, most of the current researches mainly focused on the oil and gas flow problems inside the lubricated bearing under the ring, such as the distribution of lubricating oil in the bearing or the thickness of the oil film. Few studies have explored the coupling analysis of lubricating oil flow and heat transfer under-race lubrication. The specific law between bearing temperature and lubricating oil distribution is not very clear.

In this paper, the numerical simulation method is used to establish a threedimensional high-speed ball bearing ring lubrication analysis model. The VOF model is used to track the gas-liquid interface, to reveal the coupling relationship between internal flow and heat transfer in the bearing, and to explore the coupling of rotational speed and oil supply to the internal flow-temperature field of the bearing. Finally, for the cooling effect of the bearing outer ring, the definition of oil penetration rate in the under-ring lubrication method is put forward quantitatively, the lubrication mechanism of the outer ring is revealed, which deepens the understanding of bearing internal lubrication performance. It has practical guiding significance for the design and optimization of the bearing lubrication system.

\section{Numerical Calculation Method}

\subsection{Mathematical Model}

Since the oil and gas are in a two-phase stratified flow state in the high-speed ball bearing, the oil and gas are incompatible with each other and the basic fluid equations are satisfied between the phases [9]. Therefore, this paper uses the VOF model [10] to calculate the two-phase flow field inside the bearing and track the position change of the oil-gas interface. The $\varphi_{\text {oil }}$ is used to represent the volume fraction of the oil phase in each unit volume, $\varphi_{\text {air }}$ is used to represent the volume fraction of the gas phase in each unit volume, and the sum of the two-phase volume fraction of oil and gas in each control unit is 1 . The two-phase volume fraction of oil and gas meets:

$$
\varphi_{\text {oil }}+\varphi_{\text {air }}=1
$$

It means that the lubricating oil and air are conserved in the calculation unit, and $\varphi_{\text {oil }}$ can be calculated by the volume fraction equation.

$$
\frac{\partial}{\partial t}\left(\varphi_{\text {oil }} \rho_{\text {oil }}\right)+\nabla \cdot\left(\varphi_{\text {oil }} \rho_{\text {oil }} v\right)=S_{\alpha_{\text {oil }}}
$$

In the formula: $\rho_{\text {oil }}$ represents the density of the oil phase, and $v$ is the velocity vector, which $S_{\alpha_{\text {oil }}}$ represents the generalized source term for the quality of lubricating oil.

For oil and gas two phases, the continuity equation 


$$
\nabla \cdot(\rho \boldsymbol{v})=0
$$

Momentum equation

$$
\nabla \cdot(\rho \boldsymbol{v} \boldsymbol{v})=-\nabla \cdot p+\rho \boldsymbol{g}+\boldsymbol{F}+\nabla \cdot\left(\mu\left(\nabla \boldsymbol{v}+(\nabla \boldsymbol{v})^{T}\right)\right)
$$

Energy equation

$$
\nabla \cdot[\boldsymbol{v}(\rho E)]=\nabla \cdot\left(k_{f} \nabla T\right)
$$

In the formula: $\rho$ is the pressure, $\boldsymbol{g}$ is the acceleration of gravity, $\boldsymbol{F}$ is the external force term, $T$ is the temperature, and $E$ is the energy.

The PISO algorithm is used to solve the problem, and the pressure term is PRESTO! The format, considering that the cage, rolling elements, and inner ring of the angular contact ball bearing drive the airflow in the cavity when the angular contact ball bearing is running at high speed, the bearing cavity is in a high-speed motion turbulent state [3], the turbulence model selects the RNG $k-\varepsilon$. The model can describe the high-speed and vortex flow phenomenon well [11]. RNG $k-\varepsilon$ model is a classical turbulence model for high-speed rotating or swirling flow. It considers the influence of high strain rate, large curvature, and other factors on the calculation, and can improve the accuracy under rotating flow. It is suitable for the study of oil and gas two-phase flow of high-speed bearing, which is well documented in a series of papers on high-speed bearing published by Wu et al [12-13].

MRF (Multiple Reference Frame) can assign different rotational and translational velocities to an independent computational region, so as to solve the motion of multiple rotating bodies with different velocities. The applicable scope of the MRF is mostly used for the calculation of time-averaged flow.

\subsection{Calculation Model}

Figure 1 is the geometric model of M50 all-steel angular contact ball bearing, and its dimensions are shown in table 1. Eight under-ring holes are distributed at equal intervals in the upper circumferential direction of the inner ring. Under the combined action of centrifugal force and pressure, lubricating oil enters the bearing cavity through these small holes to realize under-ring lubrication.

The computational domain is divided into two parts: fluid domain and solid domain. The fluid domain includes under-ring holes, internal flow field, and external flow field. The internal flow field is located between the inner and outer rings and is the main area where fluid interacts with the rolling elements; the external flow field is located on both sides of the bearing end face and is connected to the atmosphere, and is used to simulate the process of two-phase flow of oil and gas in and out of the bearing; the solid domain is mainly considered fluid-solid coupling heat transfer. At the same time, due to the complexity of the internal structure of the bearing, a hybrid grid is used. 


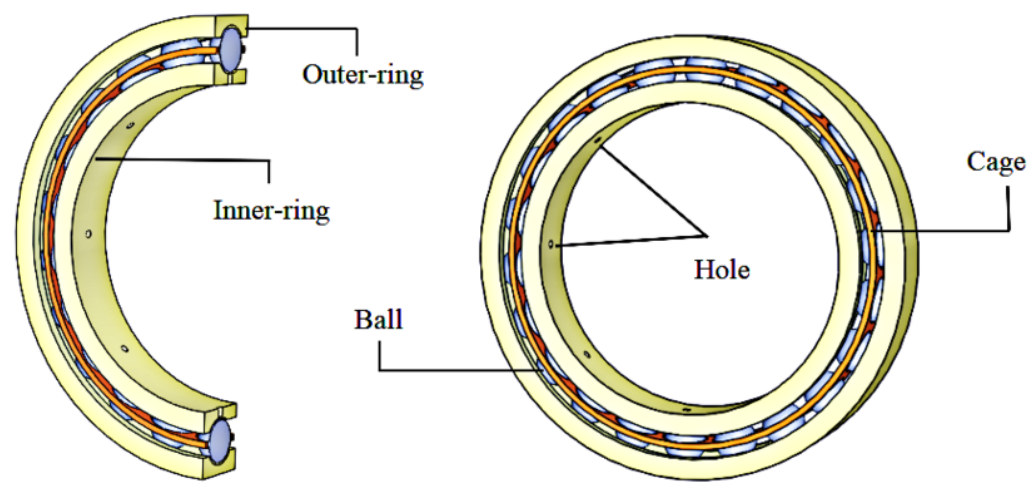

Figure 1. Geometry model.

Table 1. Structural parameters.

\begin{tabular}{ccccccc}
\hline $\begin{array}{c}\text { Inner } \\
\text { diameter } \\
(\mathbf{m m})\end{array}$ & $\begin{array}{c}\text { Outer } \\
\text { diameter } \\
(\mathbf{m m})\end{array}$ & $\begin{array}{c}\text { Width } \\
(\mathbf{m m})\end{array}$ & $\begin{array}{c}\text { Ball } \\
\text { diameter } \\
\mathbf{( m m})\end{array}$ & $\begin{array}{c}\text { Number } \\
\text { of balls } \\
/\end{array}$ & $\begin{array}{c}\text { Contact } \\
\text { angle } \\
(\mathbf{d e g})\end{array}$ & $\begin{array}{c}\text { Diameter of oil } \\
\text { supply hole under } \\
\text { the inner ring } \\
(\mathbf{m m})\end{array}$ \\
\hline 150 & 213 & 30 & 20.24 & 22 & 33.75 & 2.8 \\
\hline
\end{tabular}

Both the fluid domain and the solid domain are unstructured grids with high accuracy. The mesh sizes of the two regions are the same, and the mesh quality of the whole model is greater than 0.6 . The operating parameters selected for grid independence verification are: speed $8000 \mathrm{r} / \mathrm{min}$, oil supply $10 \mathrm{~L} / \mathrm{min}$, axial load $19.6 \mathrm{kN}$, radial load $2.1 \mathrm{kN}$. The temperature of the bearing inner ring and outer ring simulated under four different grid numbers are compared. It can be seen from figure 2 that when the mesh number reaches 980000 , the temperature of the bearing inner ring will not change; when the grid number reaches 1880000 , the temperature of the bearing inner ring and outer ring will not change. Therefore, 1880000 grids are selected for numerical simulation analysis. The grid division is shown in figure 3.

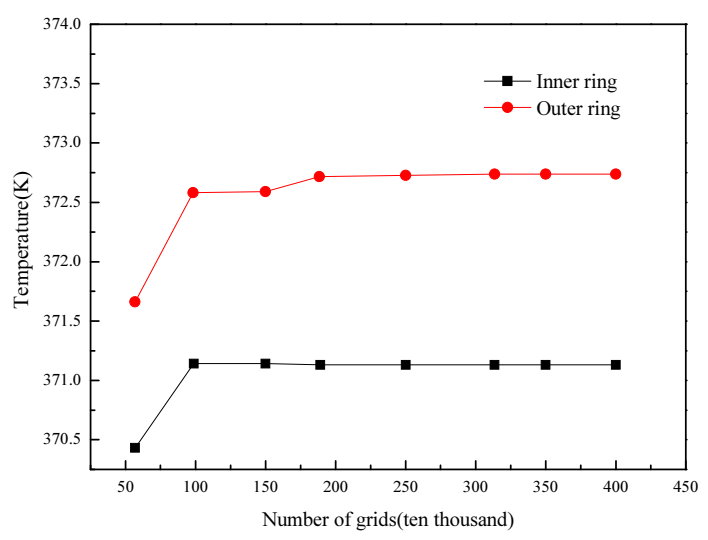

Figure 2. Grid independence verification. 


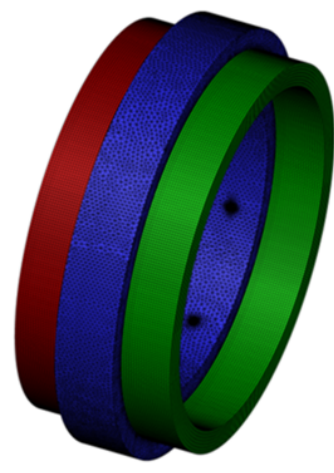

(a) Overall grid

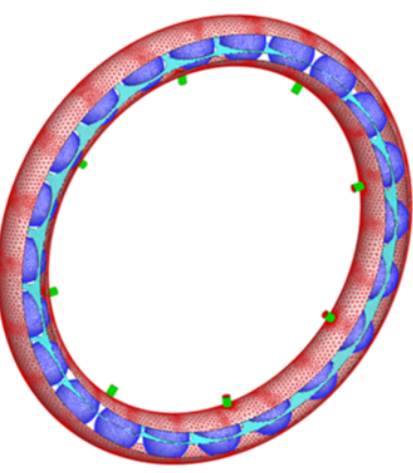

(b) Internal flow field grid

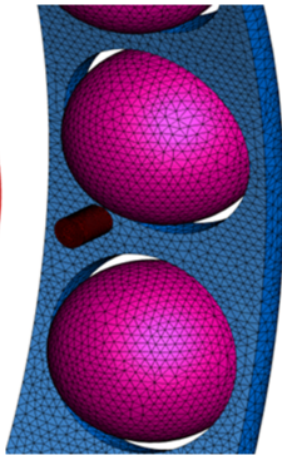

(c) Ring grid under the ball

Figure 3. Mesh of the computational domain.

\subsection{Boundary Conditions}

The motion form of high-speed angular contact ball bearing is very complex. The inner ring, cage and ball revolve at their respective speeds. The ball also has the speed of rotation in addition to revolution. Therefore, this article uses MRF to describe the motion pattern of the ball bearing. The outer ring is fixed, the inner ring and the spindle rotate at the same speed, and the speed [14] of the cage and the ball is:

$$
\begin{aligned}
& n_{b}=\frac{1}{2} n\left(1-\frac{D_{b} \cos \alpha}{d}\right) \\
& n_{w}=\frac{d n}{2 D_{b}}\left(1-\frac{D_{b}^{2} \cos \alpha^{2}}{d^{2}}\right)
\end{aligned}
$$

Where: $n$ is the inner ring speed, $n_{b}$ is the ball and cage revolution speed, $n_{w}$ is rotation speed of the ball, $D_{b}$ is the ball diameter, $\alpha$ is the contact angle, and $d$ is the bearing pitch circle diameter.

The inner ring of the bearing is set as the boundary of the rotating wall, the outer ring is set as the stationary wall, and the cage is set as the boundary of the wall and rotates. The heat generation rate of the bearing is obtained by Palmgren's empirical formula [15]. According to Palmgren's empirical formula, the calculated heat generation of the ball bearing is evenly distributed to the contact surface of the bearing inner ring, outer ring, and ball in the form of volume heat source. The specific details are as follows: the heat exchange between bearing inner and outer rings and lubricating oil is mainly caused by friction torque $M_{0}$ generated by lubricant viscosity and friction torque $M_{1}$ generated by load.

$$
\begin{aligned}
& M=M_{0}+M_{1}=10^{-7} f_{0}(v n)^{\frac{2}{3}} D_{m}{ }^{3}+0.0013\left(P_{0} / C_{0}\right)^{0.33} P_{1} D_{m} \\
& Q=1.05 \times 10^{-4} M n
\end{aligned}
$$

Where $M$ is the total friction torque, $M_{0}$ is the torque related to the bearing type, speed, and lubricating oil properties, and $M_{1}$ is the torque related to the load on the bearing. The heat exchange between bearing inner and outer rings and lubricating oil is mainly composed of $M_{0}$ and $M_{1} \cdot f_{0}$ is the coefficient related to bearing type and 
lubrication mode. In this paper, $v$ is the kinematic viscosity of lubricating oil, $D_{m}$ is the average diameter of bearing, $P_{0}$ is the equivalent static load of bearing, $C_{0}$ is the rated static load of bearing, $P_{1}$ is the bearing load.

It is uniformly distributed to the contact surface between the inner and outer rings of the bearing and the ball in the form of a volume heat source. Eight holes distributed in the circumferential direction of the bearing inner ring are set as mass flow inlets. At the same time, to prevent backflow, the axial ends are set as pressure outlets, and the pressure is a standard atmospheric pressure. The multiple reference frame (MRF) is used to simulate the relative speed between different parts of the bearing [16]. The residual convergence thresholds of turbulent kinetic energy, turbulent dissipation, and energy of each iteration are all set to $10^{-3}$, and the rest are set to $10^{-5}$, the average temperature of the outlet lubricating oil is detected, and the temperature is considered to be stable when the temperature difference between the inlet and outlet of the lubricating oil changes within $1 \%$ in 5000 iteration steps; meanwhile, the mass flow at the inlet and outlet boundary is detected, and the calculation convergence is considered when the net flux is less than $3 \%$.

\subsection{Boundary Conditions}

The experimental data in the literature[17] is selected for verification, and the verification results are shown in figure 5. Both simulation and experiment conditions are axial load $19.6 \mathrm{kN}$, radial load $2.1 \mathrm{kN}$, lubricating oil inlet temperature $353 \mathrm{~K}$, inlet flow rate $15 \mathrm{~L} / \mathrm{min}$. In this paper, the commercial software Fluent 19.0 is used to study the problem of high speed bearing oil-gas two-phase flow.

According to figure 4, it can be seen that the simulation value and the experimental value change with the increase of speed basically the same, the calculation error of the inner ring temperature is within $0.5 \%$, and the outer ring is within $1 \%$. It can be seen that the calculation model in this paper can be used to study the two-phase flow of oil and gas inside the bearing.

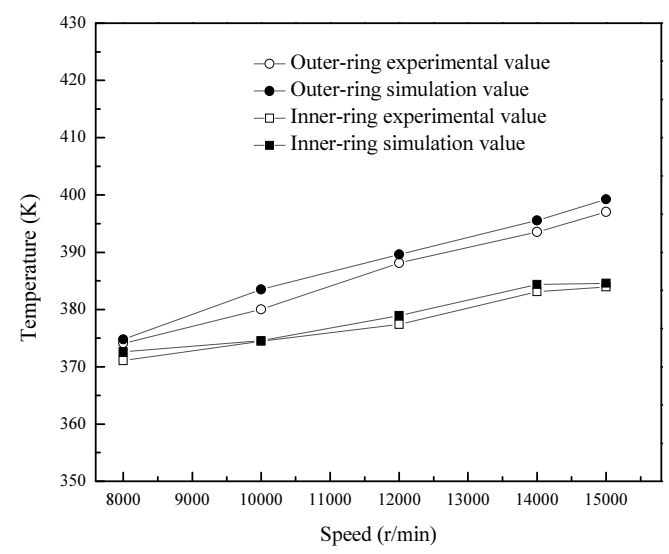

Figure 4. Temperature verification of inner and outer rings (oil supply: $15 \mathrm{~L} / \mathrm{min}$, radial load: $2.1 \mathrm{kN}$, axial load: $19.6 \mathrm{kN})$.

According to table 2, the average temperature rise errors of the inner and outer rings are $-5.38 \%$ and $7.84 \%$ respectively, which indicates that the numerical model can be used to study the oil-gas two-phase flow in the bearing. 
Table 2. Physical parameters of oil and gas.

\begin{tabular}{cccc}
\hline & $\begin{array}{c}\text { Experimental value of } \\
\text { temperature rise } \\
(\mathbf{K})\end{array}$ & $\begin{array}{c}\text { Simulated value of } \\
\text { temperature rise } \\
(\mathbf{K})\end{array}$ & Error (\%) \\
\hline Inner ring & 10.03 & 9.49 & -5.38 \\
Outer ring & 17.08 & 15.74 & 7.84 \\
\hline
\end{tabular}

\section{Results and Analysis}

\subsection{Analysis of Two-phase Flow Field and Temperature Field in Bearing}

The working conditions of $8000 \mathrm{r} / \mathrm{min}$ and $15 \mathrm{~L} / \mathrm{min}$ of oil supply is selected to analyze the two-phase flow field of lubricating oil under the high-speed ball bearing ring and the temperature field of the inner and outer rings. In order to further explore the reasons for the difference in oil distribution on the inner and outer ring surfaces, as shown in figure 5 (a), the upper and lower radial sections of the bearing are selected along the Z-X direction, and the velocity field of the radial section is analyzed, as shown in figure 5 (b) and figure 5(c), it can be seen that the movement of the fluid inside the bearing is bounded by the cage, and there are obvious differences. The flow velocity between the outer ring and the cage is large, but the velocity gradient is small; the velocity distribution between the inner ring and the cage is just the opposite. The high-speed flow causes a large shear force near the outer ring, which reduces the adhesion of the lubricating oil to the outer ring, and most of it is thrown out from the end surface. At the same time, the existence of the cage also prevents the lubricating oil from penetrating the outer ring. Based on this analysis, it is concluded that the lubrication effect of the outer ring of the bearing is poorer than that of the inner ring under the under-ring lubrication method.

Figure 6 shows the temperature distribution and azimuth angle of the inner and outer rings of the bearing. It can be seen from figure 6(a) that the average temperature of the inner ring is lower than the average temperature of the outer ring. This is because the amount of oil on the inner ring surface is lower than the outer ring surface. The amount of lubricating oil is large, and the cooling effect is obvious, which is consistent with the conclusion drawn from the above flow field. It can be seen from figure 6(b) that the temperature of the inner ring of the bearing is periodic in the circumferential direction, and the temperature of each hole under the ring is the lowest. With the hole under the ring as the periodic center, the surrounding temperature gradually increases, forming a high-temperature circular area. According to the bearing structure, heat is mainly generated at the contact and friction part of the ball and the raceway, and the presence of the ball will inhibit the spread of the oil, reduce the cooling effect, and cause the appearance of high temperature areas. Combining figure 6(a) and figure 6(b), it can be seen that the temperature of the inner ring is not uniformly distributed in the axial direction. The temperature of the large end surface is high and the temperature of the small end surface is low. This is because the large end surface of the inner ring is located at the shoulder of the bearing. It is much higher than the lower hole of the ring, and the structural reason of the high position makes it difficult for the lubricating oil to fully lubricate the shoulder of the bearing, resulting in a higher temperature on the large end of the bearing inner ring. 


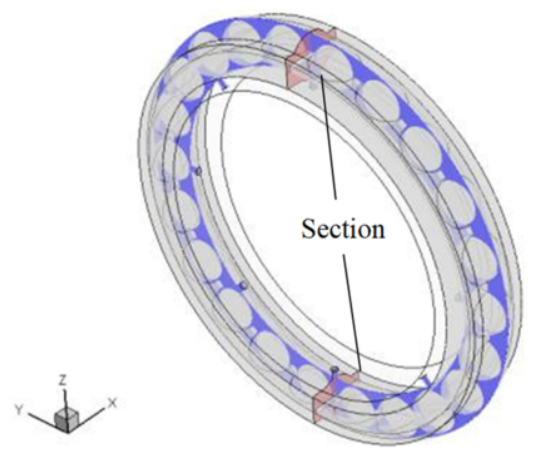

(a) schematic diagram of the internal flow field in the $\mathrm{z}-\mathrm{x}$ direction.

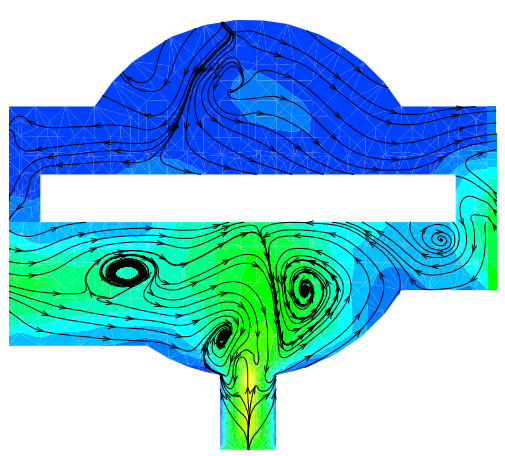

(b) Velocity cloud picture of the upper Z-X section

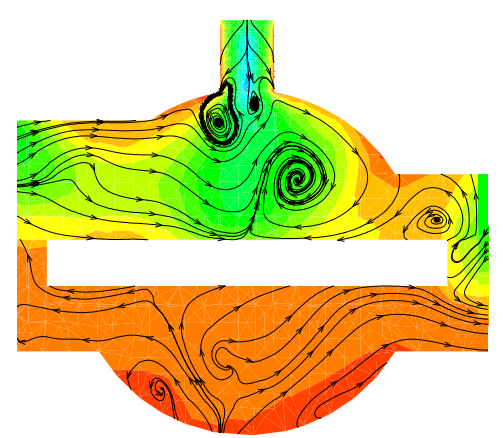

(c)Velocity cloud picture of the below Z-X section.

Figure 5. Z-X section velocity $(\mathrm{m} / \mathrm{s})$ cloud picture of internal flow field (rotating speed:8000r/min, oil supply:15L/min).

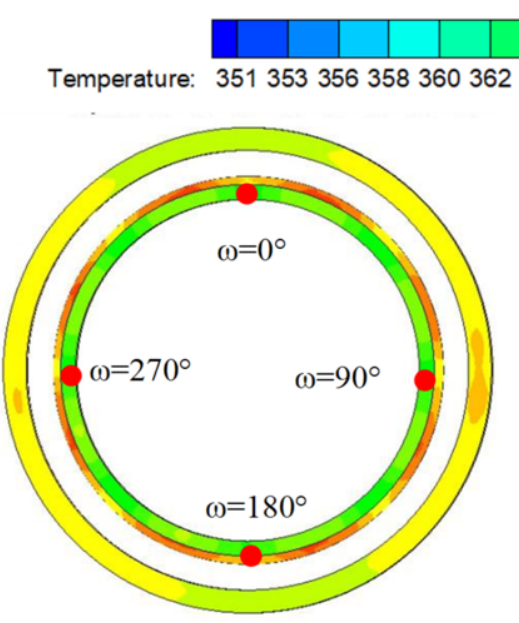

(a) Lateral temperature cloud picture of bearing

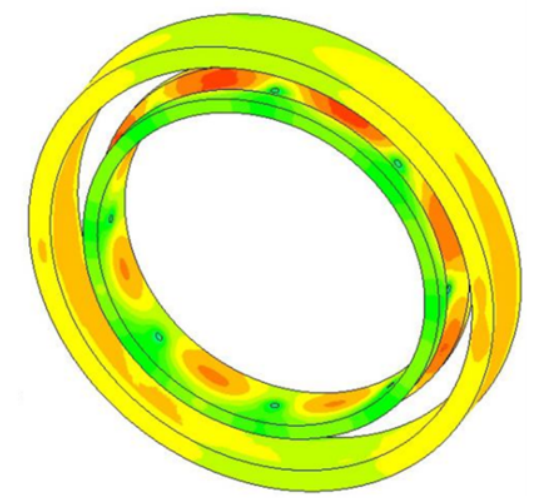

(b) Shaft temperature cloud picture of bearing

Figure 6. Cloud picture of oil volume fraction between bearing rings (rotating speed:8000r/min, oil supply:15L/min). 
Figure 7 shows that there is an obvious correlation between the oil and gas distribution and temperature distribution in the internal flow field of the lubricated bearing under the ring. Since the heat transfer performance of lubricating oil is significantly better than that of air, the convective heat transfer effect is better. The lower temperature always occurs in the place where the volume fraction of lubricating oil is large. This conclusion is consistent with the results obtained by Wu et al [13]. It indicated that there is a certain similarity between under-race lubrication and injection lubrication.

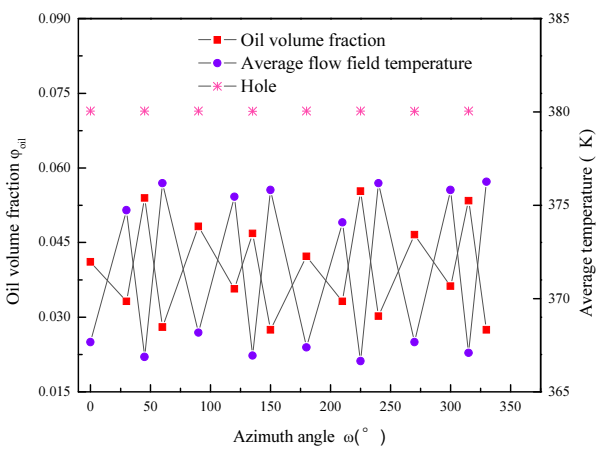

(a) Average temperature distribution of inner ring

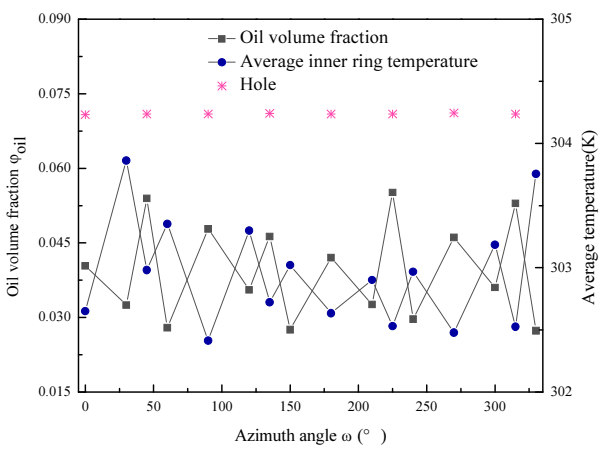

(b) Average temperature distribution of flow field.

Figure 7. Distribution of circumferential average temperature and oil volume fraction (rotating speed: $8000 \mathrm{r} / \mathrm{min}$, oil supply: $15 \mathrm{~L} / \mathrm{min})$.

\subsection{Influence of Speed}

From the analysis of figure 8(a), the distribution of the oil phase volume fraction inside the bearing is relatively regular in the circumferential direction. The oil phase volume fraction is the largest at the under-race hole, and the oil phase volume fraction between the two under-race holes is the smallest. However, with the continuous increase of the speed, the average volume fraction of the oil phase inside the bearing gradually decreases, and the difference between the maximum value and the minimum value of the oil phase volume fraction in the circumferential direction gradually decreases, and the uniformity of the distribution will gradually increase. This shows that the continuous increase of the speed will promote the distribution of lubricating oil on the inner and outer rings of the bearing to continuously approach.

Figure 8(b) and figure 8(c) shows that the continuous increase of the speed makes the overall temperature of the inner and outer rings of the bearing gradually increase. The main reason for the rise of the overall temperature of the inner and outer rings of the bearing is the increase in heat generation and the decrease of the oil volume generated by high speed. Further analysis shows that the temperature distribution of the outer ring of the bearing in the circumferential direction at the same speed is more even than that of the inner ring. This is because the amount of lubricating oil thrown to the outer ring is uniform under the action of centrifugal force. When the speed is further increased to $14000 \mathrm{r} / \mathrm{min}$ and $15000 / \mathrm{min}$, the temperature difference between the inner and outer rings is not much different and is the smallest in the research range. This shows that increasing the speed has positive significance for reducing the temperature difference between the inner and outer rings of the bearing. According to previous reports, bearing life is an inverse function of the temperature difference between the inner and outer rings [18], 
and the reduction of the temperature difference between the inner and outer rings is beneficial to improve bearing life.

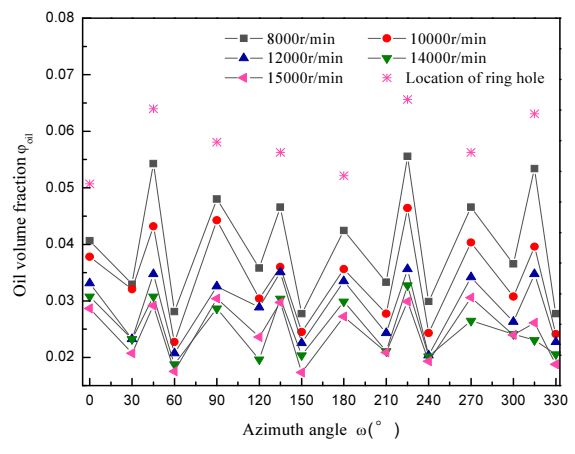

(a) Circumferential oil volume fraction

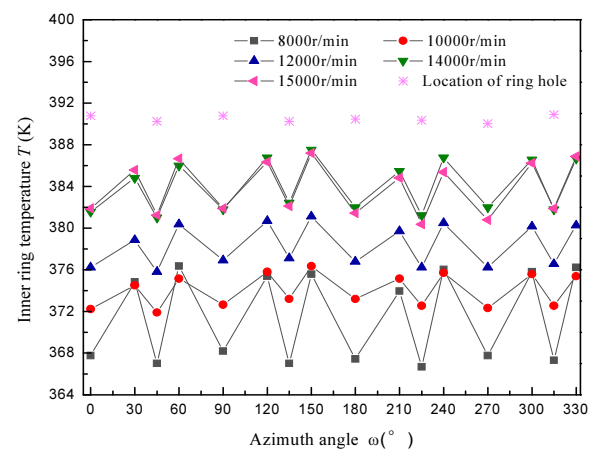

(b) Circumferential inner-ring temperature

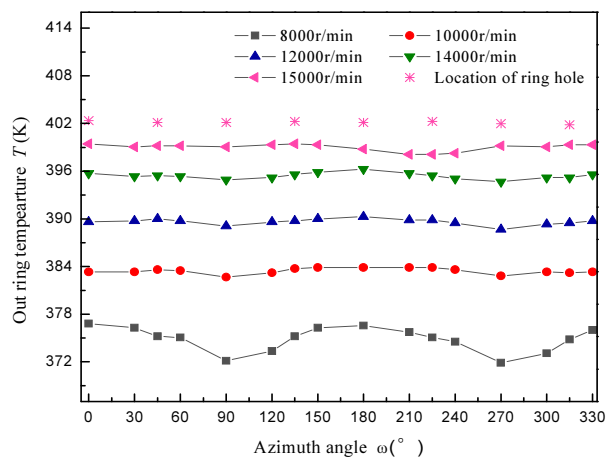

(c) Circumferential outer-ring temperature distribution

Figure 8. Volume fraction and temperature distribution of bearing circumferential oil (oil supply: 15L/min).

\subsection{Lubrication Penetration Rate Under the Ring}

According to the analysis of the first two sections, it is found that the cooling effect of the outer ring of the bearing is related to whether the lubricant can reach the surface of the outer ring, which is consistent with the conclusion of Liu et al [19-20]. Based on the above, this article proposes the definition of penetration rate to quantitatively characterize the lubrication efficiency of the outer ring of high-speed ball bearings under the under-ring lubrication mode.

In shown in figure 9, the total oil mass in the bearing is calculated according to the oil distribution diagram.

$$
m=m_{1}+m_{2}+m_{3}+m_{4}+m_{5}+m_{6}
$$

Where $\mathrm{m}$ is the total mass of lubricating oil, $\mathrm{m} 1$ is the mass of lubricating oil in the internal fluid area, $\mathrm{m}_{2}$ is the mass of lubricating oil thrown out of the bearing (left basin), $\mathrm{m}_{3}$ is the mass of lubricating oil thrown out of the bearing (right basin), and $\mathrm{m}_{4}$ is the 
ring For the oil quality of the lower hole, $\mathrm{m}_{5}$ is the oil quality of the inner ring surface, and $\mathrm{m} 6$ is the oil quality of the outer ring surface.

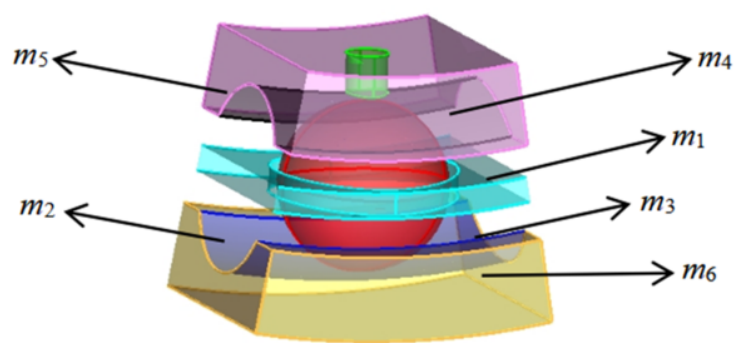

Figure 9. Distribution of lubricating oil on the bearing.

The lubricating oil quality of each part is calculated according to the oil volume fraction in the six parts. The definition of the two-phase mass in the volume fraction equation in the VOF model is introduced. The calculation formula for the lubricating oil quality of each part is

$$
m_{i}=V_{i}\left(\phi_{\text {oil }} \rho_{\text {oil }}+\left(1-\phi_{\text {oil }}\right) \rho_{\text {air }}\right) \phi_{\text {oil }}
$$

Where $V_{i}$ is the volume of each control body, $\rho_{\text {air }}$ is the air density, and $\rho_{\text {oil }}$ is the oil density.

The ratio of the mass of lubricating oil reaching the surface of the outer ring to the total mass of lubricating oil is defined as the penetration rate of lubricating oil, denoted as $\eta$, and its mathematical expression is

$$
\eta=\frac{m_{6}}{m} \times 100 \%
$$

According to the geometric dimensions of the bearing, the volume of the control body of each part is calculated, as shown in table 3 .

Table 3. Control volume of each part $\left(\mathrm{mm}^{3}\right)$.

\begin{tabular}{lccccc}
\hline $\begin{array}{l}\text { Internal } \\
\text { fluid } \\
\text { volume } \mathbf{V}_{\mathbf{1}}\end{array}$ & $\begin{array}{c}\text { Left to draw } \\
\text { volume V2 }\end{array}$ & $\begin{array}{l}\text { Right to draw } \\
\text { volume } \mathbf{V}_{\mathbf{3}}\end{array}$ & $\begin{array}{c}\text { Hole below } \\
\text { the ring } \\
\text { volume } \mathbf{V}_{\mathbf{4}}\end{array}$ & $\begin{array}{c}\text { Inner-ring } \\
\text { surface control } \\
\text { body volume } \mathbf{V}_{\mathbf{5}}\end{array}$ & $\begin{array}{c}\text { Outer-ring } \\
\text { surface control } \\
\text { body volume } \mathbf{V}_{\mathbf{6}}\end{array}$ \\
\hline 20102 & 52398 & 35796 & 114 & 2524 & 3158 \\
\hline
\end{tabular}

According to the definition of penetration rate, the oil penetration rate under different working conditions above is calculated to further explore the influence of speed and oil supply on bearing lubrication performance. It can be seen from the analysis of figure 10 that with the continuous increase of speed, the penetration rate of lubricating oil is continuously decreasing, and the penetrating ability of lubricating oil is negatively correlated with the speed. With the continuous increase of oil supply, the penetration rate of lubricating oil is increasing, and the penetration ability of lubricating oil is positively correlated with the oil supply. By comparing the penetration rate of lubricating oil at various speeds as shown in figure 10(a) and figure 10(b), the penetration rate of lubricating oil under different oil supply has obvious advantages. So in future research, the oil supply can be appropriately increased to obtain a better cooling effect when cooling the bearing through the outer ring. 


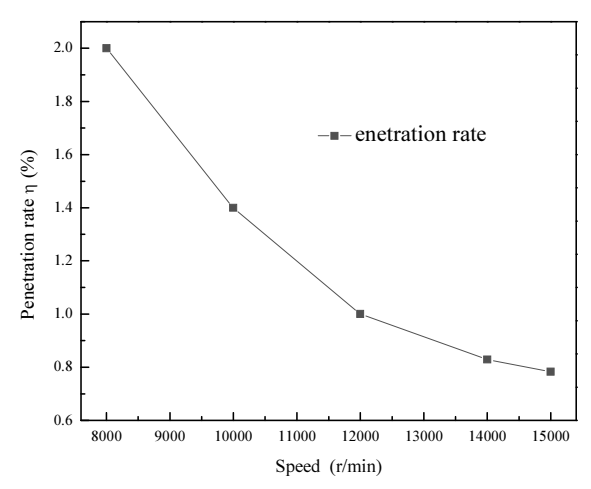

(a) Penetration rate of different speed

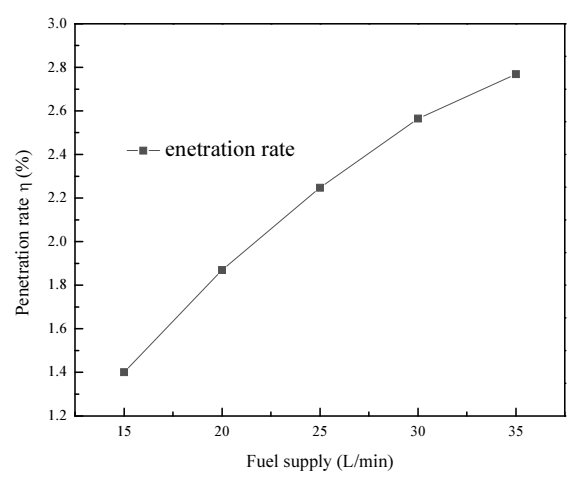

(b) Penetration rate of different oil supply

Figure 10. The penetration rate of oil in different working conditions.

\section{Conclusions}

In regards to the problem of under-ring lubrication of high-speed ball bearings, this paper analyzes the distribution characteristics of oil and gas two-phase and temperature in the bearing under different working conditions based on the VOF numerical model. From the perspective of penetration rate, it reveals the effect of under-ring lubrication efficiency with bearing operating parameters. The law of change provides an idea for future research on bearing lubrication efficiency. The specific conclusions are as follows:

- Combining the oil and gas two-phase flow field and temperature field, it can be seen that the oil and gas distribution and temperature distribution in the lubricated bearing under the ring are not uniform. The lubrication effect of the outer ring of the bearing is poorer than that of the inner ring because of the high position of the bearing, the inner ring exists a phenomenon that the temperature of the large end surface is higher than that of the small end surface should be considered in the design and optimization of the bearing lubrication system.

- The temperature of the inner and outer rings of the bearing increases with the increase of the rotating speed, and the oil volume fraction decreases with the increase of the rotating speed, but the uniformity of the distribution of both the inner and outer rings of the bearing will be improved; the increase of the rotating speed will make the distribution of the lubricating oil on the inner and outer rings of the bearing close to each other, which is of positive significance for reducing the temperature difference between the inner and outer rings of the bearing.

- Through simulation calculation, the definition of penetration rate is proposed, which is negatively correlated with the speed and positively correlated with the oil supply, which can be used to quantitatively reflect the lubrication efficiency of the outer ring under the ring. At the same time, the better lubrication and cooling effect of the outer ring of the bearing can be obtained by properly increasing the oil supply compared with reducing the rotational speed. 


\section{References}

[1] Zheng DZ, Gu L, Wang LQ et al. Effect of lubrication oil supply parameters on the performance of highspeed rolling bearing J. Journal of Harbin Institute of Technalogy,2006,38: 11-14

[2] Lin JS, Zhang ZB. Prospects of Aeroengine Power Transmission System in the 21 st Century J. Journal of Aerospace Power, 2001, 16(2): 108-114.

[3] Yan K, Dong L, Zheng JH, et al. Flow performance analysis of different air supply methods for high speed and low friction ball bearing J. Tribology International, 2018, 121: 94-107.

[4] Zhu WB, Zhang XB, LU YS, et al. Numerical study for influence of lubrication methods on lubrication performance of ball bearing. J. Journal of Propulsion Technalogy, 2019, 40(4): 892-901.

[5] Schuller FT, Signer HR. Performance of Jet-and Inner-Ring-Lubricated 35-Millimeter-Bore Ball Bearings Operating to 2.5 Million DN R. NASA-TP-1808, 1981.

[6] Schuller FT, Pinel SI, Signer HR. Effect of Two Inner-Ring Oil-Flow Distribution Schemes on the Operating Characteristics of a 35-Millimeter-Bore Ball Bearing to 2.5 Million DN R. NASA-TP2404,1985 .

[7] Signer HR, Bamberger EN, Zaretsky EN. Parametric Study of the Lubrication of Thrust Loaded 120mm Bore Ball Bearings to 3 Million DN R. NASA-TM-X-68264,1973.

[8] Zhai Q, YAN K, Zhang YY, et al. Investigation of air flow pattern and heat transfer efficiency inside cavity of high-speed angular contact ball bearing. J. Journal of Xi' an Jiaotong University, 2014, 48(12): 29-33, 40.

[9] Li XX, Yan K, Ge LF, et al. Research on flow field characteristics of oil injection lubrication for highspeed ball bearings. J. Journal of Xi' an Jiaotong University, 2019, 53(12): 17-24.

[10] Hirt C, Nichol B. Volume of fluid (VOF) method for the dynamics of free boundaries. J. Journal of Computational Physics, 1981, 39(1): 201-225.

[11] Xiao JL, Zhu EQ, Wang GD. Numerical simulation of emergency shutdown process of ring gate in hydraulic turbine runaway. J. Journal of Fluid Engineering, 2012, 134(12): 123501.

[12] $\mathrm{Wu} \mathrm{W}, \mathrm{Hu} \mathrm{CH}, \mathrm{Hu} \mathrm{JB}$, et al. Jet cooling for rolling bearings: Flow visualization and temperature distribution. J. Applied Thermal Engineering, 2016, 105: 217-224.

[13] $\mathrm{Wu} \mathrm{W}, \mathrm{Hu} \mathrm{CH}$, Hu JB, et al. Jet cooling characteristics for ball bearings using the VOF multiphase model. J. International Journal of Thermal Sciences, 2017, 116: 150-158.

[14] Harris TA, Kotzalaas RM. Rolling Bearing Analysis. M. London:Taylor and Francis Press, 2006.

[15] Wang CS. Analysis Method of Rolling Bearing. M. Beijing: Mechanical Industry Press, 1987.

[16] Kerdouss F, Bannari A, Proulx P. CFD modeling of gas dispersion and bubble size in a double turbine stirred tank. J. Chemical Engineering Science, 2006, 61(10): 3313-3322.

[17] Shoda Y, Ijuin S, Aramaki H, et al. The Performance of a Hybrid Ceramic Ball Bearing Under High Speed Conditions with the Under-Race Lubrication Method. J. Tribology Transactions, 1997 40(4): 676684.

[18] Pinel SI, Signer HR, Zaretsky EV. Comparison Between Oil-Mist and Oil-Jet Lubrication of HighSpeed,Small-Bore,Angular-Contact Ball Bearings. J. Tribology Transactions, 2008, 44(155): 327-338.

[19] Liu HB, Wang HY, Zhang L, et al. Analysis on penetration mechanism of oil jet lubrication for high speed rolling bearing. J. Journal of Aerospace Power, 2016, 31(7): 1766-1776.

[20] Liu HB, Li YB, Liu GP. Numerical investigation of oil spray lubrication for transonic bearings. J. Journal of the Brazilian Society of Mechanical Sciences and Engineering, 2018, 40(80): 1-14. 